\title{
A contribuição dos Institutos Federais para o desenvolvimento local: Uma análise comparativa para municípios do estado de Minas Gerais, Brasil
}

\author{
The contribution of Federal Institutes to local development: A comparative analysis for
} municipalities in the state of Minas Gerais, Brazil

La contribución de los Institutos Federales al desarrollo local: Un análisis comparativo para los municipios del estado de Minas Gerais, Brasil

Recebido: 20/11/2021 | Revisado: 25/11/2021 | Aceito: 26/11/2021 | Publicado: 07/12/2021

\author{
Nuno Álvares Felizardo Júnior \\ ORCID: http://orcid.org/0000-0001-7200-1289 \\ Instituto Federal Sudeste de Minas Gerais, Brasil \\ Universidade Federal de Viçosa, Brasil \\ E-mail: nuno.felizardo@ifsudestemg.edu.br \\ Marco Aurélio Marques Ferreira \\ ORCID: https://orcid.org/0000-0002-9538-1699 \\ Universidade Federal de Viçosa, Brasil \\ E-mail: marcoufv1@gmail.com \\ Rodrigo Gava \\ ORCID: https://orcid.org/0000-0002-1855-5034 \\ Universidade Federal de Viçosa, Brasil \\ E-mail: rgava@ufv.br
}

\begin{abstract}
Resumo
Uma política pública brasileira de educação voltada para o fomento do desenvolvimento local são os Institutos Federais de Educação, Ciência e Tecnologia (IF's), que apresentam uma organização descentralizada e localizada em diversos municípios, inclusive, com população abaixo de 10.000 habitantes. Essa estrutura descentralizada - multicampi - e a clara definição do escopo geográfico dos IF’s enfatizaram o compromisso de intervir em suas respectivas localidades e regiões, por meio da criação de soluções técnicas e tecnológicas que levem ao desenvolvimento sustentável com inclusão social. Portanto, entender se os Institutos Federais afetam positivamente o desenvolvimento econômico local permite convergir esforços para melhorar esse relacionamento. Isso permite a elaboração da seguinte questão: $O$ estabelecimento de Institutos Federais influencia o desenvolvimento local? A fim de responder a essa questão, foi realizado um clusterização dos municípios por meio de indicadores de desenvolvimento coletados no ano de 2008 e, posteriormente, uma análise qualitativa entre duas cidades, por meio de indicadores coletados no ano de 2016, sede e não sede de Institutos Federais. O resultado sugere que a influência dos Institutos Federais pode ser observada com mais destaque em municípios de menor concentração populacional, bem como, com mais ênfase nos indicadores de educação e, em seguida, trabalho e renda.
\end{abstract}

Palavras-chave: Institutos Federais; Desenvolvimento econômico; Políticas públicas; Análise comparativa.

\begin{abstract}
A Brazilian public policy on education aimed at promoting local development are the Federal Institutes of Education, Science and Technology (IF's), which have a decentralized organization and located in several municipalities, including those with a population of less than 10,000 inhabitants. This decentralized structure - multicampi - and the clear definition of the geographical scope of the FIs emphasized the commitment to intervene in their respective locations and regions, through the creation of technical and technological solutions that lead to sustainable development with social inclusion. Therefore, understanding whether the Federal Institutes positively affect local economic development allows us to converge efforts to improve this relationship. This allows for the elaboration of the following question: Does the establishment of Federal Institutes influence local development? In order to answer this question, a clustering of municipalities was carried out using development indicators collected in 2008 and, subsequently, a qualitative analysis between two cities, using indicators collected in 2016, headquarter and non-headquarters of Federal Institutes. The result suggests that the influence of the Federal Institutes can be observed more prominently in municipalities with a lower population concentration, as well as, with more emphasis on education indicators, followed by work and income. Keywords: Federal Institutes; Economic development; Public policy; Comparative analysis.
\end{abstract}




\begin{abstract}
Resumen
Una política pública brasileña de educación orientada a promover el desarrollo local son los Institutos Federales de Educación, Ciencia y Tecnología (IF), que tienen una organización descentralizada y están ubicados en varios municipios, incluidos aquellos con una población de menos de 10.000 habitantes. Esta estructura descentralizada multicampi - y la clara definición del ámbito geográfico de las IF enfatizaron el compromiso de intervenir en sus respectivas localizaciones y regiones, a través de la creación de soluciones técnicas y tecnológicas que conduzcan al desarrollo sostenible con inclusión social. Por tanto, entender si los Institutos Federales inciden positivamente en el desarrollo económico local nos permite converger esfuerzos para mejorar esta relación. Esto permite la elaboración de la siguiente pregunta: ¿El establecimiento de Institutos Federales influye en el desarrollo local? Para responder a esta pregunta, se realizó una agrupación de municipios utilizando indicadores de desarrollo recolectados en 2008 y, posteriormente, un análisis cualitativo entre dos ciudades, utilizando indicadores recolectados en 2016, sede y no sede de Institutos Federales. El resultado sugiere que la influencia de los Institutos Federales se puede observar de manera más prominente en los municipios con menor concentración poblacional, así como, con mayor énfasis en los indicadores de educación, seguidos del trabajo y los ingresos.
\end{abstract}

Palabras clave: Institutos Federales; Desarrollo económico; Políticas públicas; Análisis comparativo.

\title{
1. Introdução
}

O Instituto de Pesquisa Econômica Aplicada (IPEA), no ano de 2017, por meio de seu estudo Desenvolvimento Regional no Brasil - Políticas, estratégias e perspectivas, destaca o esforço de investimentos públicos federais em inovação para contribuir no combate ao status quo de reprodução da dependência e do atraso das periferias nacionais e da continuidade da preservação da hegemonia de interesses oligárquicos tradicionais nesses territórios que concentram a extrema pobreza do Brasil (Monteiro Neto et al., 2017).

Nesse documento, um dos esforços para o desenvolvimento econômico brasileiro é identificado em ações como a criação dos Institutos Federais (IF’s). Os IF’s são um projeto nacional de desenvolvimento que, em sua concepção, viabilizam sistemas regionais de inovação, com o objetivo de levar a produção de conhecimento e tecnologias para locais com deficiência em seu desenvolvimento econômico, essa descentralização visa fomentar atividades inovadoras nas periferias nacionais. Com isso, essa política pública fortalecem as redes nacionais de ensino superior e ensino médio profissionalizante - por meio da expansão dos institutos federais de educação, ciência e tecnologia e das universidades federais - pode constituir uma estratégia promissora para o desenvolvimento regional brasileiro (Monteiro Neto et al., 2017).

Este contexto vai ao encontro do estudo de fomentado pelo Centro de Gestão e Estudos Estratégicos do IPEA quando sugerem novas agendas para as políticas públicas no Brasil devem, sempre que possível, ser concebidos de forma a contribuir para o combate à desigualdade. (Mazzucato \& Penna, 2016, p. indefinida)

No entanto, ressalta-se que as novas políticas industriais e tecnológicas voltadas para a promoção do desenvolvimento não substituem as ações de apoio público à infraestrutura científica e tecnológica. Ao contrário, torna-se ainda mais importante o fortalecimento das instituições de ensino e pesquisa, dentro de uma estratégia de planejamento de longo prazo (Antonelli, 2009; Cassiolato \& Lastres, 2001; Cassiolato \& Lastres, 2002; Cassiolato et al., 2003).

Portanto, analisar como uma política pública desenhada para estimular o desenvolvimento econômico se faz importante para continuidade ou reorganização de suas diretrizes. Neste artigo, esse apoio público elaborado para incentivar o desenvolvimento econômico são os IF's que, por seu turno, se baseiam na transferência de conhecimento para gerar riqueza e fomentar impactos sociais que influenciam na minoração das desigualdades sociais e majoração da inclusão social.

Esse desenho de pesquisa é respaldado pela relevância que os processos de criação, manutenção e compartilhamento da informação podem ser influenciados e dirigidos com a implantação de mecanismos de governança do conhecimento (Foss, 2007).

Para a escolha de regiões periféricas ao conhecimento, este trabalho, fundamentou-se no pensamento de Cassiolato e Lastres $(2001 ; 2002 ; 2010)$, que afirmam ser relevante o entendimento da realidade, de uma região que sinaliza desigualdade e atraso de desenvolvimento através do estudo da geração de inovação.

Corroborando com essa relevância, vários estudos recentes se esforçam para analisar a influência de instituições 
educacionais em regiões periféricas, como nos trabalhos de Pereira e Cardoso (2020), Lins (2021), Debastiani et al. (2018), Dantas e Guenther (2021), Costa et al., (2017) e Sousa e Freiesleben (2018).

Assim sendo, essa estrutura resulta no seguinte problema de pesquisa: H1 - Há evidências de influência dos Institutos Federais no desenvolvimento local, mais especificamente, em municípios do estado de Minas Gerais?

Diante dessa inquietação, objetiva-se identificar municípios hospedeiros dos IF’s, no estado de Minas Gerais, classificando-os por meio da formação de Clusters e analisar suas influências em indicadores de desenvolvimento local.

Em relação aos objetivos específicos, foram definidos: A). Explicar o que são os IF'se como estão constituídos; B). Realizar um mapeamento através de indicadores de desenvolvimento, e; C). Analisar a influência dos IF's sobre o desenvolvimento local.

Destarte, o artigo se organiza da seguinte forma: primeiro é apresentada a orientação teórica, depois seu desenho metodológico, os resultados e sua discussão e, por fim, a conclusão.

\section{Referencial Teórico}

Como referência teórica este trabalho lançou mão das teorias desenvolvimentistas atuais, identificadas por Castelo (2012): macroeconomia estruturalista do desenvolvimentismo (Bresser-Pereira e Gala, 2010); pós-keynesiana, e; socialdesenvolvimentista, bem como as teorias seminais de desenvolvimento regional de Perroux e Freitas (1967), Boudeville (1970), Myrdal (1957), Hirschman (1958) adotados para estudo, respectivamente, do desenvolvimentismo e desenvolvimento local nesse artigo.

\subsection{Desenvolvimento Econômico}

O conceito de "desenvolvimento geograficamente desigual" é um construto teórico embutido dentro da análise econômica espacial e do discurso (Buscher \& Arsel, 2012). Como argumentado por Harvey (2006; 2011), representa um elemento-chave na perpetuação do capitalismo e está associado a uma dinâmica que gera privilégio seletivo a determinados espaços econômicos.

A distribuição espacial desigual da atividade econômica no Brasil permaneceu concentrada em uma região central do estado de São Paulo, começando com o período de industrialização no início do século XX até os dias atuais (Furtado, 1977; Prado Júnior, 1981; Prado Júnior \& Fernandes, 2000), permanecendo até os estudos mais recentes onde alertam que 56 cidades brasileiras detêm $47 \%$ da renda (IPEA, 2010).

Devido a essa concentração, pesquisas sobre modelos de desenvolvimento periféricos são particularmente importantes para países como o Brasil, onde a distribuição espacial desigual da atividade econômica tem sido particularmente marcante e tem persistido ao longo do tempo (Krugman, 1999).

O reconhecimento dessa realidade despertou renovado interesse em políticas de desenvolvimento regional e local e em conceituações de "novo regionalismo" que fundamentam o novo pensamento sobre o espaço e o desenvolvimento econômico (Rogerson, 2009; Yeung, 2009).

Assim, a análise do desenvolvimento é tomada como processo e envolve a mobilização de recursos materiais e humanos, dentro de um território definido onde encontramos a infraestrutura material os recursos naturais e a organização sociopolítico-cultural (Santos, 2000).

As concepções e enfoques do desenvolvimento são diversos e tem mudado ao longo do tempo, entretanto, parece comum a diversas correntes de pensamento a ideia de que o desenvolvimento se fundamenta em dois princípios, a sustentabilidade e a endogeneização (Dallabrida, 2010). 
O desenvolvimento econômico convencional tem como desígnio ser atrativo para empresas já estabelecidas externamente o que faz com que sua chegada resulte na majoração da renda média, porém reiteradamente é negligente quanto à proteção ambiental ou à equidade social. Em contra partida, quando o processo de desenvolvimento econômico propõe a participação da comunidade resulta na diversificação da economia - por exemplo: cluster de negócios, zonas de tecnologia -, intensificação das relações sociais, qualificação de mão-de-obra e serviços sociais entre regiões - por exemplo: treinamento de gestão, condições de saneamento básico - além de desenvolvimento de indústrias verdes e ambientalmente amigáveis - por exemplo: programa de eficiência energética, incentivos de construção verde (Grodach, 2017).

Nessa concepção o desenvolvimento não significa apenas crescimento econômico, mas relações harmônicas entre a economia, o ambiente e a sociedade com a participação das pessoas no processo e criação de uma identidade regional e espírito de pertencimento (Arocena, 1997).

De fato, a natureza territorial - ou local - do desenvolvimento econômico há muito tempo conhece uma situação de marginalidade teórica. No entanto, a crise do modelo fordista de produção em massa facilitou a redescoberta teórica de formas de produção flexível em nível local (Albuquerque, 2004). Esse movimento abre o caminho para uma maneira diferente de interpretar a realidade e, especialmente, a realidade territorial, da qual a proposta de desenvolvimento endógeno surge (Ramírez, 2006).

A dinâmica produtiva atual baseada nas constantes mudanças em inovação e tecnologia resulta em possibilidades objetivas e subjetivas para o território repensar o papel que os fatores endógenos podem desempenhar na busca de soluções para do desenvolvimento (Ramírez, 2006).

Antes de qualquer reflexão que vise abordar problemas específicos, como é o caso, é importante destacar que “o desenvolvimento endógeno é o tratamento da definição mais ampla do termo Desenvolvimento, que deve corresponder a uma abordagem objetiva e realista para o mesmo" (Alemán, 2006, p. 115).

Por isso, quando se considera as tendências ou as propostas para o desenvolvimento, que tem como escopo o combate às questões inerentes ao desnível social e o desprezo pelo aspecto ambiental dos países periféricos, destaca-se o lócus da dimensão local sob o argumento de que nela estariam presentes aspectos que, por envolver aspectos culturais locais, distanciam de uma simples reprodução das determinações globais que reestruturam a ordem econômica mundial (Arocena,1988).

Não é necessário identificar, então, o desenvolvimento econômico local como se fosse um modelo de industrialização pós-fordista oposto ao funcionamento da grande empresa. De fato, e, para esta pesquisa, ponto fulcral, o foco do desenvolvimento econômico local "enfatiza fundamentalmente os valores territoriais, identidade, diversidade e flexibilidade que existiram no passado nas formas de produção não baseadas apenas na grande indústria, mas em características gerais e locais de um território determinado" (Albuquerque, 2004, p. 158).

Assim, a principal causa da marginalização teórica da natureza territorial do desenvolvimento econômico deve ser buscada na simplificação desse processo evolutivo que fez muito do pensamento econômico deixasse a referência territorial e tomar, unicamente, como unidade de análise a empresa ou o setor econômico, considerado abstratamente, isto é, desconectado de seu ambiente territorial. Dessa forma, o território foi reduzido a um espaço uniforme e indiferenciado e, por muito tempo, a análise central do desenvolvimento econômico permaneceu dominada pelo conceito de economias de escala internas à empresa, aspecto que tem sido compartilhado pelas principais linhas do pensamento econômico (Albuquerque, 2004).

Outra consequência dessa abordagem analítica é a redução da visão de desenvolvimento econômico para o caminho do desenvolvimento industrial que se baseia na grande empresa verticalmente integrada e está ligada aos processos de urbanização. Essa forma de raciocínio, ainda bem presente, foi qualificada, no entanto, pela contribuição de Alfred Marshall (1890), que no final do século XIX, ao examinar a concentração geográfica da indústria, propôs como unidade de estudo do desenvolvimento econômico como parte da base territorial (Albuquerque, 2004). 
A teoria da organização industrial Marshall e seu conceito de organização - que não pode ser reduzida apenas ao empreendedorismo - dão, portanto, uma chave teórica fundamental para a abordagem do desenvolvimento econômico local, para restabelecer o território como unidade de análise. Esta teoria marshalliana de organização industrial é oposta, portanto, para a teoria de localização industrial de raiz neoclássica como o papel central da empresa no último é substituído em que pelo ambiente local e a agregação ou aglomeração (aglomerado) onde a empresa está localizada. Do mesmo modo, as economias internas de escala ligadas à dimensão empresarial são acompanhadas pelas economias externas geradas pelas interdependências locais (Albuquerque, 2004).

Neste recorte teórico, destaca-se os três atores principais no desenvolvimento econômico local que são: "o governo local, os negócios locais e as comunidades locais, conhecidos como triângulo do desenvolvimento econômico local (DEL)" (World Bank, 2016, p. 1). Esses atores devem ter uma visão e trabalhar juntos para alcançar o sucesso no desenvolvimento econômico.

\subsection{Instituto Federal de Educação, Ciência e Tecnologia}

Nesse contexto do DEL emerge uma nova realidade de suporte aos centros periféricos com a ação expansionista da educação politécnica dos últimos 10 anos por meio dos Institutos Federais de Educação, Ciência e Tecnologia (IF's) - unidade de análise do artigo.

Devido ao recente esforço de capilarização da Rede Federal de Educação Profissional e Tecnológica - Rede Federal , que se iniciou com a lei de criação dos Institutos Federais em dezembro de 2008, são poucas as pesquisas sobre a atuação dessas instituições e, inexistentes, quando relacionada à efetiva ação sobre o desenvolvimento local.

Essa marginalização da promoção dos IF's dificulta o entendimento de sua atuação em relação a algumas de suas finalidades e objetivos que foram concebidos, dentre os quais se destacam:

“Art. 6을 Ostitutos Federais têm por finalidades e características:

[...]

II - desenvolver a educação profissional e tecnológica como processo educativo e investigativo de geração e adaptação de soluções técnicas e tecnológicas às demandas sociais e peculiaridades regionais;

[...]

VII - desenvolver programas de extensão e de divulgação científica e tecnológica;

Art. 7o Observadas as finalidades e características definidas no art. 6o desta Lei, são objetivos dos Institutos Federais: I - ministrar educação profissional técnica de nível médio, prioritariamente na forma de cursos integrados, para os concluintes do ensino fundamental e para o público da educação de jovens e adultos;

II - ministrar cursos de formação inicial e continuada de trabalhadores, objetivando a capacitação, o aperfeiçoamento, a especialização e a atualização de profissionais, em todos os níveis de escolaridade, nas áreas da educação profissional e tecnológica;

III - realizar pesquisas aplicadas, estimulando o desenvolvimento de soluções técnicas e tecnológicas, estendendo seus benefícios à comunidade;

IV - desenvolver atividades de extensão de acordo com os princípios e finalidades da educação profissional e tecnológica, em articulação com o mundo do trabalho e os segmentos sociais, e com ênfase na produção, desenvolvimento e difusão de conhecimentos científicos e tecnológicos;

$\mathrm{V}$ - estimular e apoiar processos educativos que levem à geração de trabalho e renda e à emancipação do cidadão na perspectiva do desenvolvimento socioeconômico local e regional;

[...]" (Brasil, 2008, p. indefinida).

Para alcance do desenvolvimento regional, Myrdal (1957) afirma que é necessário desenvolver práticas inovadoras através de pesquisa e extensão - lócus de atuação dos IF’s. Pois, além da promoção de conhecimento, através do ensino técnico, de graduação e pós-graduação, os IF's lançam mão em sua infraestrutura de elementos que promovem interação com a sociedade para geração de inovações, cursos de educação para jovens e adultos para além da idade escolar e, também, projetos de extensão particularmente voltados para a consciência ambiental e social da comunidade inserida. 
Com esse objetivo, de fomentar o desenvolvimento local por meio da ação de uma organização educacional como norte, a presente pesquisa se propõe a analisar seu alcance durante uma década de atuação seguindo a metodologia a seguir.

\section{Metodologia}

Os aspectos metodológicos da pesquisa são apresentados no quadro 1.

Quadro 1 - Aspectos metodológicos.

\begin{tabular}{|l|l|}
\hline Abordagem & Quali-Quanti \\
\hline Quanto aos Fins & Descritiva e Exploratória \\
\hline Quanto aos meios & Técnica de métodos mistos sequenciais \\
\hline Unidades de análise & $\begin{array}{l}\text { Dos } 853 \text { municípios do estado de Minas Gerais somente municípios com mais de 200.000 habitantes foram } \\
\text { desconsiderados, descartando os municípios sedes de Universidades Federais. } \\
\text { Após a primeira triagem, 738 mostraram dados consistentes sobre os indicadores de desenvolvimento. IFMG } \\
\text { - Campus Bambuí e Congonhas; IF Norte de Minas - Campus Salinas; IF Sudeste MG - Rio Pomba; IF Sul de } \\
\text { Minas - Campus Inconfidentes, Machado e Muzambinho. }\end{array}$ \\
\hline Coleta de Dados & $\begin{array}{l}\text { Os dados foram coletados na plataforma virtual - Índice Mineiro de Responsabilidade Social (IMRS) entre os } \\
\text { anos de 2008 e 2016. }\end{array}$ \\
\hline Análise de Dados & $\begin{array}{l}\text { Como forma de classificar os municípios, utilizou-se a técnica de análise de agrupamentos (clusters), } \\
\text { agrupando-os conforme as semelhanças e, ao mesmo tempo, separando os grupos de acordo com a } \\
\text { heterogeneidade nos indicadores supracitados (Creswell, 2010; 2015). Por fim, uma comparação qualitativa } \\
\text { dos indicadores de desenvolvimento para o ano de 2016 - os dados mais recentes durante a execução da } \\
\text { pesquisa - foi realizada para aceitar ou rejeitar a hipótese. }\end{array}$ \\
\hline
\end{tabular}

Fonte: Autores.

No intuito de verificar se os Institutos Federais têm influenciado no desenvolvimento local, perfazendo uma de suas finalidades de criação, utilizou-se inicialmente de pesquisa do tipo quantitativo-descritivo. Esse tipo de pesquisa tem por objetivo a coleta de dados de uma população ou amostra para verificação das hipóteses previamente estabelecidas (Lakatos \& Marconi, 2010).

No presente estudo, os dados foram coletados na plataforma virtual Índice Mineiro de Responsabilidade Social (IMRS). Nessa perspectiva, recolheram-se os dados dos indicadores, apresentados na Tabela 1, disponibilizados pela base do ano de 2008, ano de criação dos Institutos Federais.

Tabela 1 - Indicadores para formação de Cluster.

\begin{tabular}{|c|c|c|}
\hline GRUPO & INDICADOR & ABREVIAÇÃO \\
\hline \multirow{3}{*}{ SAÚDE } & Proporção de internações por doenças de veiculação hídrica & PIDVH \\
\hline & $\begin{array}{l}\text { Proporção de internações por doenças relacionadas ao saneamento ambiental } \\
\text { inadequado }\end{array}$ & PDVSAI \\
\hline & Proporção de nascidos vivos cujas mães realizaram 7 ou mais consultas de pré-natal & PNVM7PN \\
\hline \multirow{3}{*}{ EDUCAÇÃO } & Taxa de adolescentes que frequentam o ensino médio na série adequada & TAFEMSA \\
\hline & Índice de Qualidade Geral da Educação & IQGE \\
\hline & Taxa de Escolarização Líquida do ensino médio & TELEM \\
\hline \multirow{2}{*}{ EMPREGO E RENDA } & Rendimento médio no setor formal & RMSF \\
\hline & Taxa de emprego no setor formal & TESF \\
\hline POPULAÇÃO & População Total & PT \\
\hline
\end{tabular}

Fonte: Autores. 
A princípio realizou-se análise exploratória das 738 observações analisando a média, mediana, moda, desvio padrão, variância, valores máximos e mínimos, assimetria e curtose.

Como forma de classificar os municípios, buscou-se por meio da técnica de análise de conglomerados (clusters), agrupá-los de acordo com similaridades e, ao mesmo tempo, separar os grupos conforme a heterogeneidade nos indicadores supracitados (Corrar et al.,2007).

Por fim, realizou-se uma comparação qualitativa de indicadores de desenvolvimento do ano de 2016 - dados mais recentes quando da execução da pesquisa - para aceitar ou rejeitar as seguintes hipóteses:

H0: Não há influência dos Institutos Federais no desenvolvimento local;

H1: Há influência dos Institutos Federais no desenvolvimento local.

\subsection{Modelo Analítico}

Para melhor compreensão da proposta de análise apresenta-se, na Figura 1, o modelo analítico orientador da busca dos resultados.

Figura 1 - Modelo Analítico.

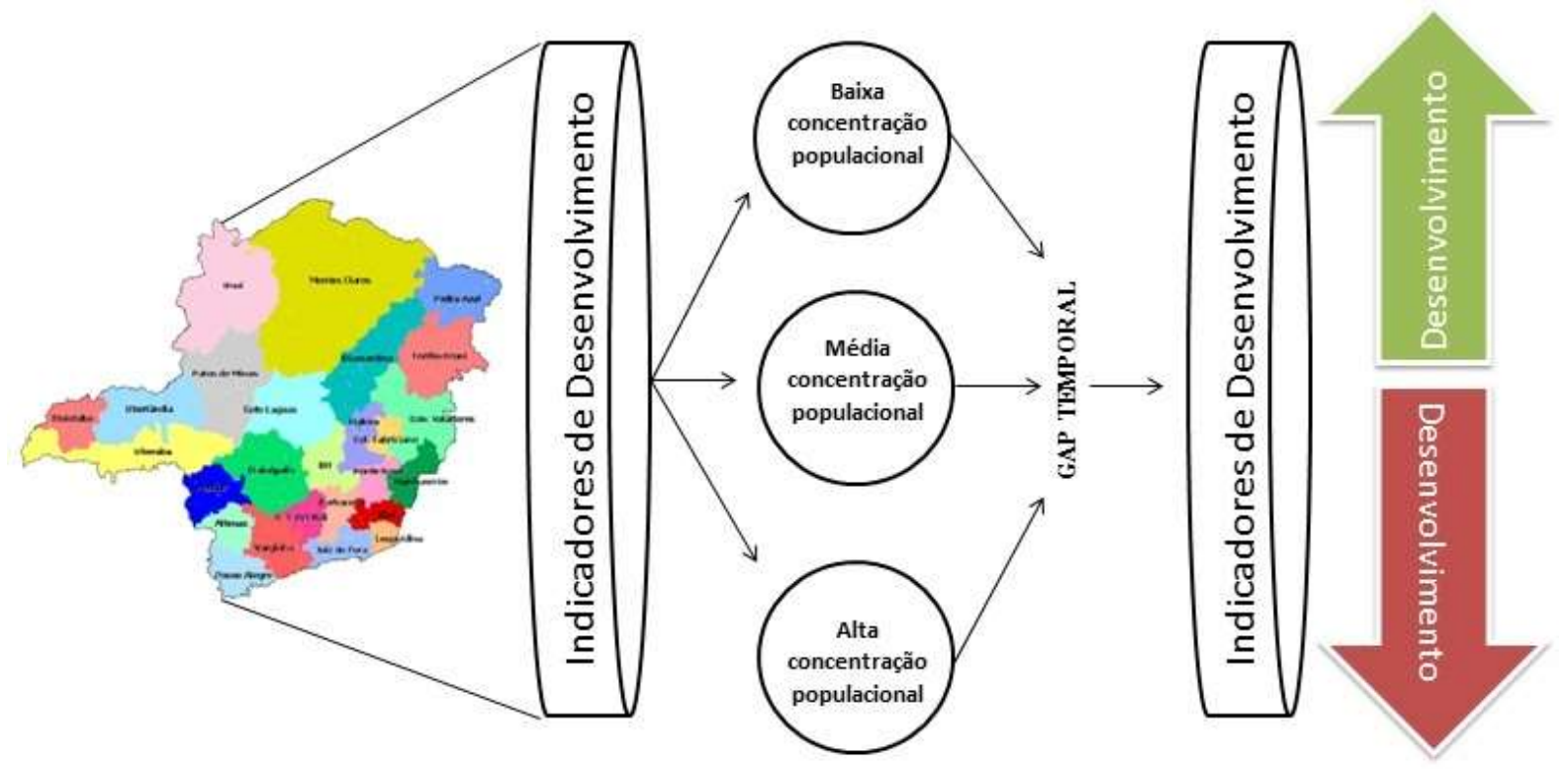

Fonte: Elaboração Própria.

\section{Análise de Dados e Discussão dos Resultados}

Para realizar os objetivos propostos pela pesquisa, fez-se a análise exploratória de todos os dados analisando as estatísticas descritivas conforme apresentadas na Tabela 2. A amostra conta com 738 observações, decorrente de 9 indicadores: 3 de saúde; 3 de educação; 2 de emprego e renda, e; 1 populacional. Em seguida, realizou-se a estatística descritiva dos munícipios conglomerados de acordo com as características de cada cluster.

\subsection{Análise exploratória dos dados}

Inicialmente realizou-se estatística descritiva dos percentuais obtidos contemplando média, desvio padrão, variância, assimetria, curtose, valores máximo e mínimo. A Tabela 2 apresenta a estatística descritiva com os indicadores de condições para desenvolvimento. 
Tabela 2 - Estatística descritiva.

\begin{tabular}{lccccccc}
\hline Indicador & Mínimo & Máximo & Média & Desvio padrão & Variância & Assimetria & Curtose \\
\hline PIDVH & 0,09 & 22,34 & 2,4953 & 2,83319 & 8,027 & 2,825 & 11,332 \\
\hline PDVSAI & 0,14 & 22,5 & 3,4006 & 3,27811 & 10,746 & 2,366 & 7,368 \\
\hline PNVM7PN & 9,8 & 100 & 58,839 & 18,0617 & 326,226 & $-0,274$ & $-0,472$ \\
\hline TAFEMSA & 9,6 & 100 & 39,846 & 11,2949 & 127,575 & 0,044 & 1,52 \\
\hline IQGE & 17 & 64 & 37,12602 & 6,985474 & 48,797 & $-0,025$ & 0,209 \\
\hline TELEM & 12 & 100 & 44,756 & 11,7291 & 137,571 & $-0,08$ & 1,466 \\
\hline RMSF & 121,02 & 2856,13 & 830,5417 & 237,8063 & 56551,834 & 2,317 & 11,628 \\
\hline TESF & 1,8 & 82,1 & 18,331 & 10,3076 & 106,247 & 1,234 & 2,585 \\
\hline PT & 827 & 199319 & 16904,22 & 23083,6 & 532852351 & 3,388 & 14,073 \\
\hline
\end{tabular}

Fonte: Resultado da Pesquisa.

Verifica-se que o índice de qualidade geral da educação (IQGE) variou entre 17 e 64 com média de 37,12. O município com menor IQGE foi Santo Antônio do Retiro cidade com população estimada de 6.898 no ano de 2008. Em contrapartida, dentro a amostra, encontra-se a cidade de Jacuí, de população um pouco menor, 7.484, compondo as 389 cidades que se encontram acima da média do indicador.

No que tange o rendimento médio do setor formal (RMSF), importante indicador para formação de conglomerado com mesmas características para o desenvolvimento, nota-se uma discrepância entre os valores mínimo - R\$121,02 - e máximo $\mathrm{R} \$ 2.856,13$, sendo que a cidade que apresenta o menor valor é o município de Mamonas que conta com população estimada de 6.288 situada na microrregião de Janaúba, já o maior RMSF é encontrado em uma cidade de parecido tamanho populacional 6.630 habitantes - do sudoeste de Minas Gerais, possivelmente influenciada pelas ações de Furnas já que a diferença para a cidade que exibe a segunda maior RMSF - Itapeva - é de R \$699,98. Quando se leva em consideração a média apenas 254 cidades estão acima dos R \$ 830,54 de RMSF.

Em relação à concentração populacional, com a limitação do estudo para cidades de até 200.000 habitantes, a maior cidade da amostra é Santa Luzia - 199.319 habitantes - influenciada pela proximidade com a capital mineira, já o menor município é Serra da Saudade com 827 habitantes.

\subsection{Análise de conglomerados (Clusters)}

Utilizou-se o programa SPSS para determinar o número de conglomerados que melhor agrupasse as 738 observações que fossem semelhantes entre si na condição para o desenvolvimento e, ao mesmo tempo, diferentes dos demais agrupados em outro (s) cluster(s).

Essa divisão dos clusters pode ser mais bem observada na Figura 2 que expõe a divisão territorial em diferentes cores de acordo com sua distribuição para cada um dos 3 (três) clusters elaborados, além de apontar os municípios que não foram considerados na análise por apresentar alguma inconsistência nos dados coletados. 
Figura 2 - Distribuição dos municípios em clusters.

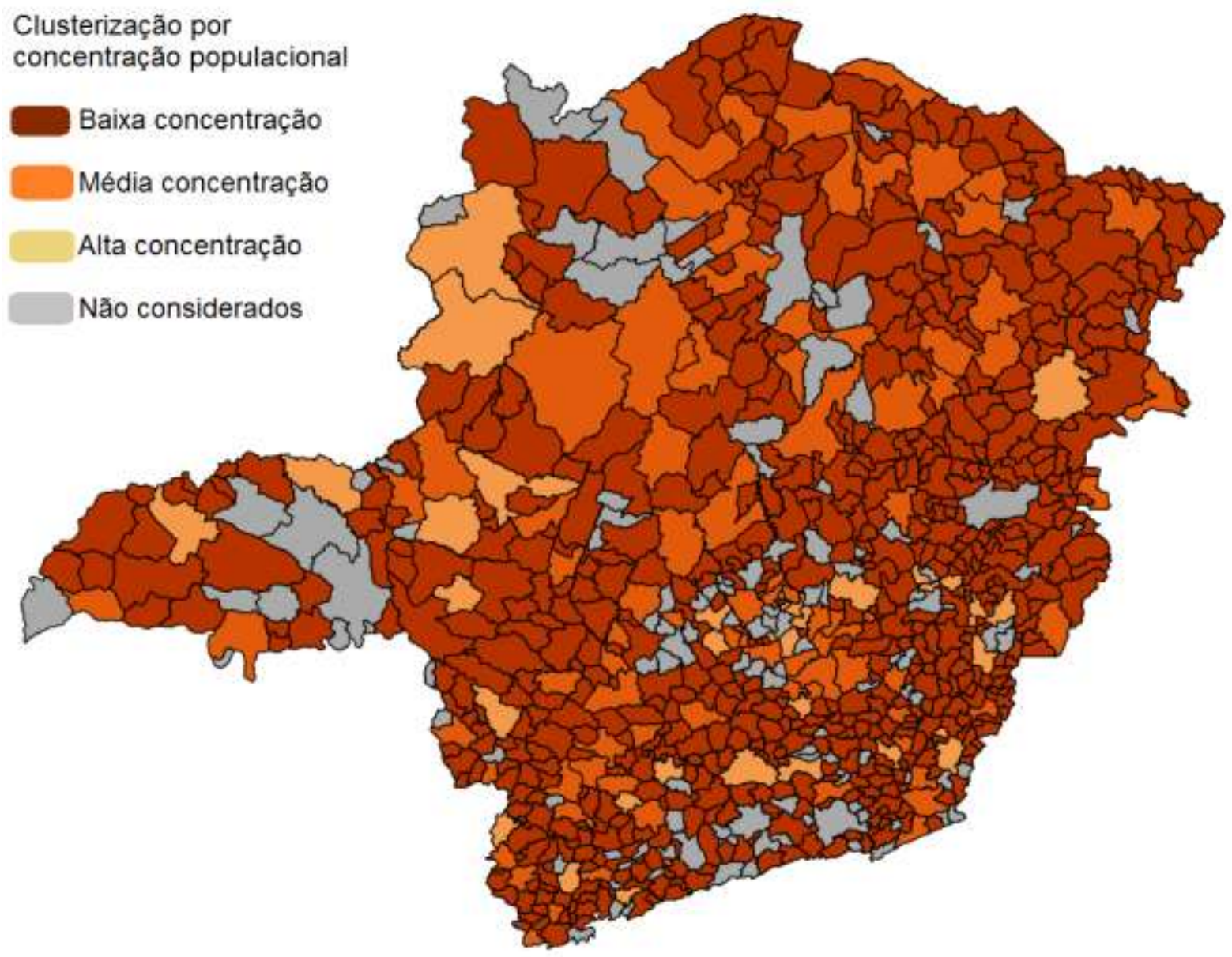

Fonte: Resultado da Pesquisa.

A divisão não apresenta concentração que mereça destaque ou justificativa. Ela segue a concentração de cidades no território Mineiro. Verifica-se, somente, uma concentração de municípios na região centro-sul do estado, importante ressaltar, que é notória a diferença de desenvolvimento no estado, apresentando, o norte do estado, histórico de desenvolvimento menor que as demais regiões.

\subsubsection{Cluster 1 - baixa concentração populacional}

O Cluster 1 é o que apresenta mais observações - 625 - evidentemente, devido a maior presença de municípios com baixa concentração populacional em Minas Gerais -, dentro dessa cluster, 4 cidades: Bambuí, Inconfidentes, Muzambinho e Rio Pomba - apresentavam Institutos Federais no ano de 2008.

Com os conglomerados constituídos, a próxima etapa é a análise qualitativa dos indicadores de desempenho entre cidades que apresentavam Institutos Federais nos anos de 2008 e 2016 e cidades que não apresentavam Institutos Federais, mas que exibiam condições para o desenvolvimento semelhantes no ano de 2008.

\subsubsection{Cluster 2 - Média concentração populacional}

O Cluster 2 é constituído por 82 observações (correspondendo a 11,11\% das observações) que apresentaram população entre 25.759 a 72.870 habitantes, o IQGE máximo é de 37,91 que se aproxima do índice apresentado por algumas cidades que apresentam Campus do Instituto Federal - Congonhas $(36,00)$ e Ouro Preto $(35,00)$ - com o mesmo índice de Congonhas também estão Pompéu, Três Pontas, Carangola, Barão de Cocais e Bocaiúva todas apresentavam IQGE de 36,00 em 2018. 


\subsubsection{Cluster 3 - Alta concentração populacional}

O Cluster 3 é composto por 31 observações (cerca de 4,2\% da amostra) que apresentaram alto índice de concentração populacional no ano de 2008. A composição desse cluster demonstra que os municípios de grande porte apresentaram condições para o desenvolvimento similares. Dos 31 municípios, 1 deles, Barbacena, sediava um Campus do Instituto Federal Sudeste de Minas Gerais.

Quanto ao RMSF, nota-se que a variância do Cluster indica que as diferenças entre os dados são baixas o que suporta sua homogeneidade, como exemplo pode comparar duas cidades de concentração populacional parecidas - Barbacena e Itaúna - enquanto a cidade de Barbacena apresentava RMSF de R\$1.126,37 a cidade de Itaúna apresentou RMSF de R\$ 1.143,63.

\subsection{Análise do desenvolvimento em cidades com e sem Institutos Federais}

O estado de Minas Gerais apresenta, em seu território, 5 (cinco) Institutos Federais de Educação, Ciência e Tecnologia contando com 53 (cinquenta e três) Campis e um parque tecnológico abrangendo em 52 diferentes cidades, distribuídos da seguinte forma: IFMG - Instituto Federal de Minas Gerais - com 17 (dezessete) Campis em 17 (dezessete) cidades diferentes; IF Triângulo Mineiro - Instituto Federal do Triângulo Mineiro - com 8 (oito) Campis, 1 (um) parque tecnológico em 7 (sete) cidades diferentes; IF Norte de Minas - Instituto Federal do Norte de Minas - com 10 (dez) Campis em 10 (dez) cidades diferentes; IF Sudeste MG - Instituto Federal do Sudeste de Minas Gerais - com 10 (dez) Campis em 10 (dez) cidades diferentes, e; IF Sul de Minas - Instituto Federal do Sul de Minas Gerais - com 8 (oito) Campis em 8 (oito) cidades diferentes.

Pela análise de Clusters, foram selecionadas as seguintes cidades por já estarem em funcionamento no ano de 2008: IFMG - Campus Bambuí e Congonhas (o Campus Ouro Preto foi desconsiderado por haver uma Universidade Federal na cidade podendo enviesar a análise); IF Triângulo Mineiro - Campus Uberaba (desconsiderado por ter população superior a 200.000 habitantes); IF Norte de Minas - Campus Salinas; IF Sudeste MG - Campus Rio Pomba e Barbacena (o Campus Juiz de Fora foi desconsiderado por ter população superior a 200.000 habitantes), e; IF Sul de Minas - Campus Inconfidentes, Machado e Muzambinho. O que resultou em um total de 8 (oito) cidades para análise de desenvolvimento.

O critério para a análise qualitativa foi a comparação entre cidades, sedes e não sedes de Institutos, que apresentavam números aproximados de população no ano de 2008, bem como, a proximidade territorial para evitar que viés de desenvolvimento regional enviesasse a análise, ou seja, apontado o Instituto dentro do Cluster, a cidade de população mais próxima, para mais ou para menos, dentro da mesma mesorregião era escolhida para análise comparativa. Neste sentido, apresenta-se, a seguir, uma comparação entre cidades de cada Cluster realizado na seção anterior.

\subsubsection{Cluster 1 - Análise qualitativa do da influência no desenvolvimento de cidades de baixa concentração populacional}

O Cluster 1 apresenta 4 (quatro) cidades sedes de Campis englobados pela delimitação supracitada: Bambuí, Inconfidentes, Muzambinho e Rio Pomba. A escolha foi entre duas cidades da Zona da Mata Mineira, pois, caso ambas sediassem Institutos, pertenceriam ao mesmo - IF Sudeste MG -, a cidade de Rio Pomba e Matias Barbosa que apresentavam, em 2008, população de 16.967 e 13.213 respectivamente, e estão a uma distância de 95 km entre elas - ver Tabela 3. 
Tabela 3 - Análise do Cluster Baixa Concentração Populacional.

\begin{tabular}{|c|c|c|c|c|c|c|c|c|c|c|}
\hline \multirow{3}{*}{2008} & Município & PIDVH & PDVSAI & PNVM7PN & TAFEMSA & IQGE & TELEM & RMSF & TESF & PT \\
\hline & $\begin{array}{l}\text { Matias } \\
\text { Barbosa } \\
\end{array}$ & 0,28 & 0,46 & 78,4 & 41,3 & 34 & 46,2 & 1023,86 & 52 & 13.213 \\
\hline & Rio Pomba & 0,17 & 1,32 & 67 & 61,8 & 36 & 69 & 1025,79 & 20,5 & 16.967 \\
\hline \multirow{3}{*}{2016} & Município & $\begin{array}{c}\text { PI } \\
\text { DVH }\end{array}$ & $\begin{array}{l}\text { PDV } \\
\text { SAI } \\
\end{array}$ & $\begin{array}{c}\text { PNV } \\
\text { M7PN }\end{array}$ & $\begin{array}{l}\text { TAFE } \\
\text { MSA } \\
\end{array}$ & IQGE & $\begin{array}{c}\text { TE } \\
\text { LEM } \\
\end{array}$ & RMSF & TESF & PT \\
\hline & $\begin{array}{l}\text { Matias } \\
\text { Barbosa } \\
\end{array}$ & 0,39 & 0,51 & 77,5 & 42,8 & 37 & 46,7 & 2031,71 & 53,8 & 14369 \\
\hline & Rio Pomba & 0,15 & 1,56 & 78,24 & 74,3 & 40 & 81,1 & 2351,44 & 27,9 & 18001 \\
\hline
\end{tabular}

Fonte: Resultado da Pesquisa.

Em relação à saúde, os dois primeiros indicadores - PIDVH e PDVSAI - é de proporção de quanto menor melhor, pois medem atendimentos médicos por veiculação hídrica e de saneamento ambiental (estando dentro do campo - Proporção de internações por condições sensíveis à atenção primária). Neste contexto, as duas cidades apresentam índices semelhantes e baixos em relação ao PIDVH, sendo que Rio Pomba conseguiu manter-se próximo a 2008, inclusive, diminuindo a proporção, no que diz respeito à PDVSAI Rio Pomba apresentou um aumento de 18,18\%. Por seu turno, Matias Barbosa registrou aumento nos dois indicadores de 39,28\% e 10,86\%, o que demonstra que houve pouca ação preventiva para conter este tipo de internação.

Já o PNVM7PN é referente ao conhecimento da necessidade da realização de pré-natal por parte da população, e a instrução emerge como ponto fundante para este indicador. Com isso, nota-se que Rio Pomba conseguiu aumentar sua proporção de atendimento em 16,77\% enquanto Matias Barbosa apresentou um pequeno decréscimo - 1,14\%.

No caso da educação, onde se espera maior influência das cidades que sediam Institutos, Rio Pomba teve um aumento nos dois indicadores de alunos matriculados no ensino médio (TELEM) 17,53\%, bem como, estes alunos estarem em série adequada 20,22\% - o que corrobora com a oferta de cursos técnicos integrados pelo Campus - e Índice de Qualidade Geral de Ensino de 11,11\%. Enquanto a cidade de Matias Barbosa registrou crescimentos mais modestos de 1,08\% (TELEM) 3,63\% (TAFEMSA) e 8,82\% (IQGE). Essa diferença já esperada pela grande atuação dos Institutos no ensino médio, técnico e tecnológico.

A influência das cidades na RMSF e TESF também apontam que a cidade de Rio Pomba prosperou mais que a de Matias Barbosa, com um aumento impactando em mais que o dobro do valor de RMSF - 129,23\% - contra um bom aumento também em Matias Barbosa - 98,43\% - importante lembrar que os valores são brutos e não houve deflação para o comparativo - o que levou a Matias Barbosa perder em poder de compra, em 2008 era de 2,47 salários mínimos e em 2016 caiu para 2,30 salários. Em relação ao número de empregados de 16 a 64 anos no setor formal, indicador que é impactado pela qualificação dos empregados nesta faixa etária, e nesta perspectiva os cursos técnicos, tecnológicos, de graduação e pós-graduação ofertados pelo Campus Rio Pomba, também podem ser vistos refletindo neste indicador já que o aumento na cidade foi de 36,09\%, enquanto Matias Barbosa, em 8 (oito) anos, foi de apenas 3,46\%.

\subsubsection{Cluster 2 - Análise qualitativa do da influência no desenvolvimento de cidades de média concentração populacional}

O Cluster 2 foi o único que o critério de mesorregião teve que ser descartado, bem como, a distância entre as cidades para que a concentração populacional não sofresse uma diferença que enviesasse a comparação. Neste sentido, optou-se por manter a proximidade populacional selecionando a cidade de Congonhas e de Guaxupé, no sul de Minas Gerais - ver Tabela 4. 
Tabela 4 - Análise do Cluster Média Concentração Populacional.

\begin{tabular}{|c|c|c|c|c|c|c|c|c|c|c|}
\hline \multirow{3}{*}{2008} & Município & PIDVH & PDVSAI & PNVM7PN & TAFEMSA & IQGE & TELEM & RMSF & TESF & PT \\
\hline & Guaxupé & 0,72 & 3,43 & 90,6 & 52,2 & 47 & 57,1 & 1045,89 & 35,4 & 48971 \\
\hline & Congonhas & 1,53 & 1,71 & 82,5 & 49,4 & 36 & 53,2 & 1374,89 & 35,4 & 46999 \\
\hline \multirow{3}{*}{2016} & Município & PIDVH & PDVSAI & PNVM7PN & TAFEMSA & IQGE & TELEM & RMSF & TESF & PT \\
\hline & Guaxupé & 1,3 & 2,57 & 93,83 & 54,5 & 43 & 57,8 & 1892,95 & 38,2 & 52108 \\
\hline & Congonhas & 0,59 & 0,25 & 88,36 & 59,1 & 46 & 64,1 & 2476,34 & 41,5 & 53348 \\
\hline
\end{tabular}

Fonte: Resultado da Pesquisa.

A evolução dos indicadores de saúde da cidade de Congonhas demonstra enorme atividade orientativa, o que pode estar relacionado aos cursos EJA do Campus Congonhas. A PIDVH teve uma redução para 38,56\% do valor de 2008, o indicador de PDVSAI acompanhou a queda vertiginosa e chegou próximo de 0 em 2016 - 0,25. Em contrapartida, a cidade de Guaxupé viu seu índice de PIDVH aumentar em 80,55\% e a PDVSAI diminuir percentualmente mas apresentar, ainda, uma proporção alta no ano de 2016.

Já em relação a PNVM7PN as duas cidades apresentaram alta proporção em 2008 acrescendo-a para o ano de 2016 . O maior aumento foi em Congonhas 7,10\% mas Guaxupé se mostra mais eficiente neste indicar - 93,83.

Os indicadores da educação sofreram maior evolução na cidade de Congonhas: TAFEMSA - aumento de 19,63\%; IQGE - um acréscimo (27,77\%) que ultrapassou o indicador da cidade de Guaxupé, e; TELEM - evolução de 20,48\%. Na cidade de Guaxupé a evolução foi mais tímida, se tratando de 8 (oito) anos de diferença, pode-se dizer que ficou inalterado o panorama da educação segundo estes indicadores e ainda houve uma retração no IQGE em $3 \%$.

Nos indicadores de emprego e renda é fácil notar uma diferença no TESF já que ambos apresentavam o mesmo indicador em 2008, ficando fácil de constatar que a cidade de Congonhas evoluiu 6,1\% enquanto Guaxupé 2,8\%. Em relação à RMSF também nota-se que apesar do aumento, Congonhas e Guaxupé perderam em poder de compra já que, Congonhas em 2008 apresentava RMSF de 3,31 salários mínimos e em 2016 de 2,81. Guaxupé também perdeu em poder de compra, em 2008 o RMSF era de 2,52 salários mínimos e em 2016 passou a ser de 2,15 salários.

\subsubsection{Cluster 3 - Análise qualitativa do da influência no desenvolvimento de cidades de alta concentração populacional}

O único município sede de um campus de Instituto Federal dentro deste cluster é Barbacena, cidade da região do Campo das Vertentes que, em 2008, tinha 123.828 habitantes. Para análise comparativa deste cluster - ver Tabela 5 - a opção foi por uma cidade da mesma região a 75 km de distância de Barbacena com população, em 2008, de 113.707 habitantes - Conselheiro Lafaiete.

Tabela 5 - Análise do Cluster Alta Concentração Populacional.

\begin{tabular}{|c|c|c|c|c|c|c|c|c|c|c|}
\hline \multirow{3}{*}{2008} & Município & PIDVH & PDVSAI & PNVM7PN & TAFEMSA & IQGE & TELEM & RMSF & TESF & PT \\
\hline & $\begin{array}{l}\text { Conselheiro } \\
\text { Lafaiete }\end{array}$ & 4,23 & 4,35 & 73,1 & 46,2 & 44 & 50,5 & 909,9 & 20,5 & 113707 \\
\hline & Barbacena & 0,26 & 0,38 & 53,9 & 60,7 & 42 & 67,3 & 1126,37 & 27,6 & 123828 \\
\hline \multirow{3}{*}{2016} & Município & PIDVH & PDVSAI & PNVM7PN & TAFEMSA & IQGE & TELEM & RMSF & TESF & PT \\
\hline & $\begin{array}{l}\text { Conselheiro } \\
\text { Lafaiete }\end{array}$ & 3,55 & 2,45 & 78,3 & 52,4 & 48 & 56,3 & 1664,33 & 23,7 & 126420 \\
\hline & Barbacena & 0,9 & 0,37 & 63,15 & 63,9 & 48 & 67,8 & 1956,51 & 28,2 & 135829 \\
\hline
\end{tabular}

Fonte: Resultado da Pesquisa. 
Os dois indicadores que devem apresentar baixos índices por sua proposta metodológica - PIDVH e PDVSAI demonstra uma forte melhora na cidade de Conselheiro Lafaiete, porém a cidade de Barbacena, desde 2008 apresenta excelentes índices nestes indicadores permanecendo próximo ao desempenho.

Em relação as consultas de pré-natal - PNVM7PN - houveram aumentos em ambas cidades, com um acréscimo percentual maior na cidade de Barbacena, 17,16\% contra 7,11\% de Conselheiro Lafaiete, no entanto Conselheiro Lafaiete já tinha um bom desempenho neste indicador no ano de 2008.

A atuação pré-existente do Campus Barbacena, por ter sido uma escola agrícola fundada no ano de 1910, indica que o esforço público advindo da mudança para Instituto Federal demonstra o aumento nos bons indicadores existentes em 2008: TAFEMSA - manteve-se próximo dos 70\%; IQGE - acréscimo de 14,28\%, e; TELEM - próximo dos $70 \%$ como o primeiro indicador analisado para educação. Em Conselheiro Lafaiete houve aumentos nos índices finítimos a 50\%: TAFEMSA - aumento de 13,46\%; IGQE - aumento de 4,34\%, e; TELEM - 5,94\% de acréscimo.

Nos indicadores de trabalho e renda, as duas cidades apresentaram desempenho similares em sua evolução.

\section{Conclusão}

O resultado das discussões sobre os indicadores coletados e as cidades componentes dos Clusters sugere algumas reflexões sobre a influência dos Institutos Federais no desenvolvimento local.

Em relação aos objetivos específicos: A) Explicar o que são os Institutos e como estão constituídos; B) Realizar um mapeamento através de formação de clusters dos IF's; C) Analisar as influências dos IF's sobre o desenvolvimento local. Eles foram atendidos nas respectivas seções: A) -2.4 ; B) -4.2 ; C) -4.3 .

Uma das finalidades dos Institutos Federais se refere ao suporte no desenvolvimento local dentro de suas características de ensino, pesquisa e extensão. Nessa perspectiva, há uma correlação quando a análise demonstra uma maior influência nos indicadores em cidades de menor concentração populacional, convergindo com objetivo de desconcentração das atividades econômicas.

Esse achado sugere a eficiência dos IF's no que tange uma de suas finalidades e características descritas pela lei 11.892 de 29 de dezembro de 2008, seção II, Art. 6º parágrafo IV: “orientar sua oferta formativa em benefício da consolidação e fortalecimento dos arranjos produtivos, sociais e culturais locais, identificados com base no mapeamento das potencialidades de desenvolvimento socioeconômico e cultural no âmbito de atuação do Instituto Federal” (BRASIL, 2008, p. indefinida).

Segundo a análise qualitativa, constata-se que a maior influência nos indicadores foi observada em cidades de menor concentração populacional, muito provavelmente atingindo outro objetivo dos IF's que é a desconcentração das atividades econômicas.

Outro destaque é para a atuação dos Institutos Federais no prisma da inclusão social, o motivo é pelas unidades de ensino ao abrangerem, além do o ensino técnico integrado ao ensino médio, a educação de jovens e adultos através da modalidade EJA. Esse esforço se mostra eficaz quando se percebe uma tendência de conscientização dos aspectos de saúde nas localidades sedes de IF maior que nos casos não sedes analisados, o que sugere que a abertura para educação de jovens e adultos pode estar influenciando na percepção de importância destes aspectos.

Por fim, respaldado pela utilização da técnica de métodos mistos, pode-se aceitar, em parte, a hipótese principal, já que: A influência dos Institutos Federais pode ser constatada com maior vigor em municípios com concentração populacional até 100.000 habitantes. Para que seja confirmada a hipótese integralmente, sugere-se um estudo de caso específico para cidades acima deste número de habitantes levando em consideração outros possíveis fatores de impulsão do desenvolvimento como Dummies. 
Outra limitação para este estudo foi o tempo de criação dos IF’s para análise de impacto no desenvolvimento, por isso a opção de analisar a influência dentro de um mesmo composto de variáveis.

Sugestões para futuros estudos são análises in loco das unidades de análise para constatação das ações que refletem nos indicadores para confirmação da atuação dos IF's em sua evolução. Outra possível pesquisa seria com egressos dos IF's para verificar a reverberação do conhecimento adquirido com os indicadores de desenvolvimento.

Análise comparativa com as demais regiões brasileiras, confirmando ou refutando os aspectos revelados pelo contexto mineiro. A utilização de indicadores de desenvolvimento por outra base ou perspectiva também seria uma opção para conflito de achados.

\section{Agradecimentos}

Agradeço ao instituto Federal do Sudeste de Minas Gerais pelo incentivo a qualificação.

\section{Referências}

Albuquerque, F. (2004). El Enfoque del Desarrollo Económico Local: Cuaderno de capacitación No. 1. Serie: Desarrollo Económico Local y Empleabilidad. Antonelli, C. (jan/jun de 2009). Economics of knowledge and the governance of commons knowledg. Revista Brasileira de Inovação, 1, 28-48.

Arocena, J. (1997). El desarrollo local frente a la globalización. Hacia un nuevo modelo de gestión local, Municipio y Sociedad Civil en Argentina, 43-58. Arocena, J. (1988). Descentralización y desarrollo local. Revista Uruguaya de Ciências Sociales, Montevideo.

Boudeville, J. R. (1970). Les spaces économiques. Paris, Boudeville, J.R.: Press Universitaires de France.

Brasil. (2008). Institui a Rede Federal de Educação Profissional, Científica e Tecnológica, cria os Institutos Federais de Educação, Ciência e Tecnologia, e dá outras providências. Lei n 11.892, de 29 de dezembro de 2008. Diário Oficial da União.

Bresser-Pereira, L. C. (2016). Reflexões sobre o Novo Desenvolvimentismo e o Desenvolvimentismo Clássic. Revista de Economia Política, $36(2), 143$.

Bresser-Pereira, L. C., \& GALA, P. (2010). Macroeconomia estruturalista do desenvolvimento. Revista de Economia Política, $30(4), 663-686$.

Büscher, B., \& Arsel, M. (2012). Introduction: neoliberal conservation, uneven geographical development and the dynamics of contemporary capitalism. Tijdschrift voor economische en sociale geografie, 103(2), 129-135.

Dependência e desenvolvimento na América Latina.

Cassiolato, J. E., \& Lastres, H. M. (2001). Arranjos e sistemas produtivos locais na indústria brasileira. Revista de economia contemporânea, 5, 103-136.

Cassiolato, J. E., \& Lastres, H. M. (2002). O enfoque em sistemas produtivos e inovação locais. Gestão do desenvolvimento e poderes locais: marcos teóricos e avaliação. Casa da Qualidade, 61-76.

Cassiolato, J. E., Lastres, H. M., \& Maciel, M. L. (2003). Pequena empresa: cooperação e desenvolvimento local.

Cassiolato, J. E., Lastres, H. M., \& Szapiro, M. (2000). Arranjos e sistemas produtivos locais e proposições de políticas de desenvolvimento industrial e tecnológico. Seminário Local Clusters, Innovation Systems and Sustained Competitiveness, IE-BNDES, Nota Técnica, 5.

Castelo, R. (2012). O novo desenvolvimentismo e a decadência ideológica do pensamento econômico brasileiro. Serviço Social e Sociedade, 112 , 613-636.

Costa, N. L., Costa, V. O., Mattos, C. A. C. de, Teixeira, O. A., Flores, A. J., \& Oliveira, G. N. de. (2017). Capital Humano e Desenvolvimento Econômico no Rio Grande do Sul: Uma Abordagem Multivariada. Desenvolvimento Em Questão, 15(38), 380-402. https://doi.org/10.21527/2237-6453.2017.38.380-402

Corrar, L. J., Paulo, E., \& Dias Filho, J. M. (2007). Análise multivariada: para os cursos de administração, ciências contábeis e economia. Atlas.

Crewell, J. W. (2010). Projeto de pesquisa métodos qualitativo, quantitativo e misto. Em J. W. CRESWELL, Projeto de pesquisa métodos qualitativo, quantitativo e misto.

Creswell, J. W., \& Clarck, V. L. (2015). Pesquisa de Métodos Mistos: Série Métodos de Pesquisa. Penso Editora.

Dallabrida, V. R. (2010). A gestão social dos territórios nos processos de desenvolvimento territorial: uma aproximação conceitual. Sociedade, Contabilidade e Gestão, 2(2).

Dantas, M. W., \& Guenther, M. (2021). University Outreach and Local Sustainable Development: A literature review. Research, Society and Development, 10(6), e23010615243. https://doi.org/10.33448/rsd-v10i6.15243

Debastiani, A., Flach, L. \& Mattos, L. (2018). Políticas Públicas para a Educação Superior e Desenvolvimento Local. Revista Gestão Universitária, 10 
Research, Society and Development, v. 10, n. 16, e99101623655, 2021

(CC BY 4.0) | ISSN 2525-3409 | DOI: http://dx.doi.org/10.33448/rsd-v10i16.23655

Foss, N. J. (2007). The emerging knowledge governance approach: Challenges and characteristics. Organization, 1, $29-52$.

Furtado, C. (1977). Prefácio anova economia politica. Editora Paz e Terra. Translated to Spanish in the same year as to Italian in.

Grodach, C. (2017). Urban cultural policy and creative city making. Cities, 68(2017), 82-91.

Harvey, D. (2006). Spaces of global capitalism. Verso.

Harvey, D. (2011). Roepke lecture in economic geography-Crises, geographic disruptions and the uneven development of political responses. Economic geography, 87(1), 1-22.

Hirschman, A. O. (1958). The strategy of economic development. New Haven: Yale University Press.

Krugman, P. (1999). The role of geography in development. International regional science review, 22(2), $142-161$.

Lakatos, E. M., \& Marconi, M. d. (2010). Fundamentos de Metodologia Científica. São Paulo: Atlas.

Lins, M. A. T. (2021). A relação entre a educação e o desenvolvimento econômico no Brasil no período de 1950 a 1970. Revista Labor, 1 (25), DOI: https://doi.org/10.29148/labor.v1i25.67957

Mazzucato, M., \& Penna, C. (2016). The Brazilian innovation system: a mission-oriented policy proposal. Brasília, DF, Brasil: Centro de Gestão e Estudos Estratégicos.

Monteiro Neto, A. O., Castro, C. N., \& Brandão, C. A. (2017). Desenvolvimento regional no Brasil: políticas, estratégias e perspectivas. Instituto de Pesquisa Econômica Aplicada (Ipea).

Myrdal, G. (1957). Economic theory and under-developed regions. Methuen.

Pereira, C. E., \& Cardoso, P. H. G. (2020). Contribuição das Instituições de Ensino Superior no processo de crescimento e desenvolvimento da Região Metropolitana do Cariri Ceará Brasil. Research, Society and Development, 9(3), DOI: http://dx.doi.org/10.33448/rsd-v9i3.2531

Prado Junior, C. (1981). O que e a liberdade: capitalismo socialismo. Brasiliense.

Prado Júnior, C. P., \& Fernandes, F. (2000). Clássicos sobre a revolução brasileira. Editora Espressão Popular.

Perroux, F., \& De Freitas, J. L. (1967). A economia do século XX.

Ramírez, F. A. (2006). Importancia de las MiPyMEs en las Aglomeraciones Empresariales. Una estrategia para el desarrollo regional en Colombia. Revista Facultad de Ciencias Económicas, 14(1), 173-186.

Rogerson, C. M. (2009). Strategic review of local economic development in South Africa. Final report submitted to Minister S Shiceka of Department of Development Planning and Local Government (DPLG). Commissioned by DPLG and GTZ.

Santos, M. (2000). Por uma outra globalização (Vol. 17). Record.

Sousa, F. E., \& Freiesleben, M. (2018). A educação como fator de desenvolvimento regional. Revista da FAE, 21(2), $163-178$.

Yeung, H. W. C. (2009). Regional development and the competitive dynamics of global production networks: an East Asian perspective. Regional studies, 43(3), 325-351. 